Diabetologe 2017 · 13:150-151

DOI 10.1007/s11428-017-0211-0

Online publiziert: 21. April 2017

C) Springer Medizin Verlag GmbH 2017

CrossMark

A. Liebl

Zentrum für Diabetes- und Stoffwechselerkrankungen, m\&i Fachklinik Bad Heilbrunn, Bad Heilbrunn, Deutschland

\title{
Typ-1-Diabetes in verschiedenen Lebensphasen
}

Mortalität unserer Patienten verbessert werden können.

Die Entwicklung auf dem Gebiet der kontinuierlichen Glukosemessung (CGM [„,continuous glucose monitoring"] und FGM ["flash glucose monitoring "]) geht so rasant voran, dass es auch technologisch interessierten Ärzten schwer fällt, Schritt zu halten. Findige Bastler unter den Menschen mit Typ-1Diabetes haben schon lange Insulinpumpen und CGM zum quasi „künstlichen“ Pankreas vereint. Gehr gelingt es, den Paradigmenwechsel in der Glukosemessung verständlich darzustellen und einen geordneten Überblick über die Vielzahl von Geräten und neue Entwicklungen mit ihren spezifischen Vor- und Nachteilen zu geben. Es kann gar nicht oft genug betont werden, dass eine strukturierte CGM-Schulung für den Erfolg dieses Verfahrens absolut unabdingbar ist. schreiben eine Vielzahl praxisrelevanter und hilfreicher Empfehlungen für die tägliche Therapie. Es ist beeindruckend, wie rasch gerade die komplexen modernen Diabetestechnologien bei den Kindern Verbreitung finden: Laut Deutscher Gesundheitsbericht Diabetes 2017 werden inzwischen $85 \%$ der Kinder unter 5 Jahren mit Insulinpumpen behandelt. Das lässt ahnen, in welche Richtung sich die Therapie der Erwachsenen in Zukunft entwickeln wird.

Siegmund $\mathrm{u}$. Thomas befassen sich mit der Insulinpumpentherapie bei Erwachsenen. Sie machen deutlich, dass diese heutzutage in hohem Maß ausgereift ist. So entwickelte sie sich mittlerweile zur verbreiteten Standardtherapie des Typ1-Diabetes. Immer mehr verdichtet sich die umfassend dargelegte Evidenz, dass mit einer optimierten Insulinpumpentherapie auch harte Endpunkte und die

\section{》) Mit der Entwicklung auf dem Gebiet der CGM und FGM ist kaum Schritt zu halten}

Trotz aller Fortschritte wird es jedoch stets einige Patienten geben, bei denen eine gestörte Insulinresorption das Problem ist. Der Beitrag über die kontinuierliche intraperitoneale Insulininfusion (CIPII) zeigt für diese schwerstbetroffenen Patienten einen alternativen Weg der Insulinapplikation auf. Die attraktiven Vorteile dieser physiologischsten aller Therapieformen müssen jedoch gegen deren erhöhte Kosten und Komplikationen abgewogen werden. Es sei in diesem Zusammenhang noch einmal betont, dass bei den meisten sog. „uneinstellbaren“ Patienten nicht Probleme der Insulinresorp- tion im Vordergrund stehen, sondern vielmehr Non-Compliance oder psychologisch-psychiatrische Störungen.

Zeyfang u. Bahrmann wenden sich zum Schluss der schnell wachsenden Gruppe älterer Menschen mit Typ-1Diabetes zu. Waren früher geriatrische Patienten mit dieser Erkrankung eine Rarität, stellen sie heutzutage einen großen Teil der ausgesprochen schwer $\mathrm{zu}$ therapierenden Menschen in der Geriatrie dar. Es ist häufig schwierig zu erkennen, wann der älter werdende Patient seine Diabetestherapie nicht mehr selbstständig durchführen kann. Zeyfang u. Bahrmann nennen einfache Tests und zeigen Hinweise auf, wie dieser wichtige Zeitpunkt erkannt werden kann und welche praktischen Schritte dann $\mathrm{zu}$ ergreifen sind.
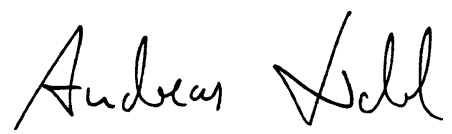

A. Liebl

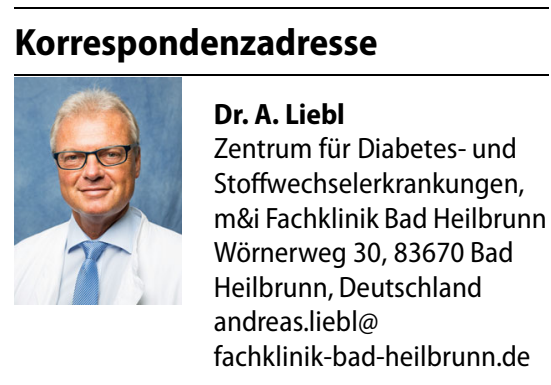

Interessenkonflikt. A. Liebl erhielt von folgenden Firmen Honorare bzw. finanzielle Unterstützung für die Teilnahme an Advisory Boards, allgemeine Beratung, ungebundene Forschungsunterstützung oder sonstige medizinisch-wissenschaftliche Leistungen: AstraZeneca, Bayer, Becton Dickinson, Boehringer-Ingelheim, Bristol Myers Squibb, DexCom, Lilly, Medtronic, MSD, Novo Nordisk, Roche, Sanofi. 
Hier steht eine Anzeige.

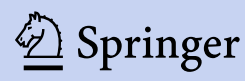

